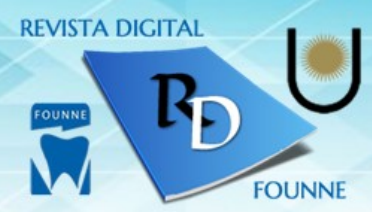

\title{
Título:
}

\section{EVALUACIÓN DE LA EFECTIVIDAD DE UNA FORMULACIÓN MAGISTRAL DE TINTURA DE PROPÓLEOS.}

Autores: Fernández V.* Rosende R.** Ortiz Barreto E.*** Briend M.****

Autor de correspondencia:fernandezvr@hotmail.com Av. Libertad 5450. CP 3400. Facultad de Odontología, Universidad Nacional del Nordeste. Corrientes, Argentina.

Recepción: 15 de mayo 2017

Aceptación: 03 de junio 2017

\section{Resumen}

Introducción. Las queilitis comisurales son reacciones inflamatorias que se manifiestan clínicamente como descamaciones, erosiones y fisuras radiadas o transversales que continúan el plano de oclusión labial con sintomatología variable y que posteriormente se extiende a la piel. El objetivo del trabajo consistió en la evaluación de la tintura de propóleos al 5\% en el tratamiento de las queilitis angulares y de la posible reacción adversa medicamentosa.

Materiales y Método. El estudio se realizó en pacientes de ambos sexos, a partir de los 15 años de edad con diagnóstico clínico de queilitis comisural. Previo consentimiento por escrito, se seleccionaron en forma aleatorizada simple para conformar dos grupos. El primer grupo, tomado como control, recibió tratamiento tradicional. El segundo grupo denominado experimental recibió la formulación objeto de estudio, la tintura de propóleos al 5\%.

Resultados. Dan cuenta de una probable preponderancia cuantitativa de la formulación objeto de estudio en comparación con la formulación tradicional de betametasona valerato a $0,1 \%$.

Conclusiones. La valoración comparativa de las evoluciones de los grupos demuestra que ambas medicaciones reducen en grado variable la intensidad de los signos y la sintomatología, sin efecto medicamentoso adverso.

\section{Palabras clave}

Inflamación, labio, citología, tópico.

\section{Autores:}

*Fernández, Víctor Ricardo. Esp. Od. Profesor Adjunto. Clínica Estomatológica. 


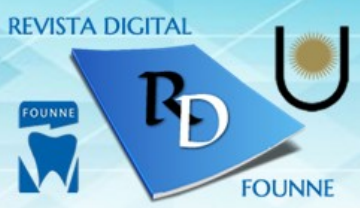

**Rosende, Roque Oscar. Dr. Profesor Titular. Cirugía III. Buco - Máxilo Facial.

*** Ortiz Barreto, Elena Soledad. Esp. Od. Auxiliar Docente. Clínica Estomatológica

****Briend María Susana. Mgter. Profesora Titular. Anatomía Patológica.

\section{Introducción}

Las queilitis son procesos inflamatorios inespecíficos de los labios que cursan con una semiología común como descamación, erosión y fisuras; dado la singularidad histológica de la zona se manifiestan desde el punto de vista terapéutico como un órgano con poca expresividad, en el sentido que causas muy diversas pueden tener las mismas exteriorizaciones clínicas. ${ }^{1 .}$

La mayor parte de las queilitis se dan en el labio inferior y quizás sea debido a una mayor exposición a los diferentes irritantes crónicos, como son luz solar, agentes mecánicos, químicos, etc. ${ }^{2}$.

Las queilitis surgen cuando a partir de una o más exposiciones de una noxa se inicia en la zona una reacción inflamatoria producida por un mecanismo inespecífico o específico generalmente manifestado como reacción alérgica. ${ }^{3 .}$

La lesión comisural se inicia con un proceso inflamatorio que posteriormente se fisura y se extiende a la piel. Las fisuras son múltiples y transversales que al infectarse son de color blanquecino, maceradas cubiertas por una capa cremosa que desaparece al frotar con una gasa, la cual deja ver una pérdida de sustancia lineal, rodeada de eritema. ${ }^{4,5,6 .}$

La irritación por el escurrimiento de la saliva es constante, aumenta consecuentemente la inflamación, es doloroso al hablar, comer y puede presentarse costras hemorrágicas. ${ }^{7}$

Se considera que la disminución de la dimensión vertical oclusiva es una causa frecuente de queilitis y en especial de la queilitis angular. ${ }^{8}$ Esta reducción de la altura facial facilita la formación de pliegues laterales profundos que son embebidos con saliva de forma constante creándose un medio con humedad propicia para la colonización por cándida albicans. ${ }^{9}, 10,11$.

Clínicamente se clasifican en agudas, subagudas o crónicas y se circunscriben a la zona comisural, se expresan mediante lesiones elementales caracterizadas por eczemas, escamas, ampollas, fisuras, erosiones superficiales que se pueden cubrir de costras y fácilmente se agregan

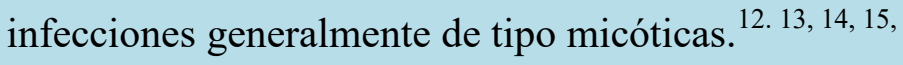




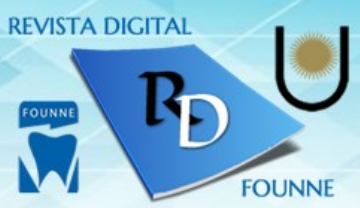

El tratamiento de las queilitis angulares consiste inicialmente en detectar el elemento causal y suprimir la noxa, aunque en ocasiones es muy difícil identificarla. En una segunda etapa se aplican antihistamínicos, corticoides, cremas de ictiol, AINEs, antimicrobianos, óleo calcáreo, antifúngicos y derivados del propóleos. 16, 17, 18. 19, 20,

El objetivo del trabajo consistió en la evaluación comparativa de la tintura de propóleos al 5\% en el tratamiento de las queilitis angulares versus la medicación tradicional de betametasona valerato a $0,1 \%$ y de la reacción adversa medicamentosa. En estudios de esta naturaleza es necesario comprobar la eficacia del medicamento en las patologías para las cuales han sido desarrolladas, la posología, el tiempo de tratamiento y el registro de las RAM que pueden surgir por el procedimiento terapéutico implementado. ${ }^{21,22.23,24,25 .}$

Materiales y Método: Es un estudio comparativo y prospectivo, la muestra se conformó con pacientes de demanda espontánea. Culminada con la etapa de revisión bibliográfica, se procedió a la búsqueda y recolección de datos, se incluyó en el estudio a pacientes de ambos sexos a partir de 15 años de edad, que presentaron alteraciones en los labios con diagnóstico clínico de queilitis angular. Previo consentimiento por escrito, se distribuyeron en forma aleatoria simple para conformar dos grupos, el primer grupo recibió tratamiento con tintura de propóleos al 5\%, indicada como la formulación objeto de estudio y se denominó grupo experimental; el segundo grupo, identificado como grupo de control y se trató con la terapéutica tradicional, en base a betametasona valerato al $0,1 \%$.

Fueron excluidos los pacientes que padecían enfermedades de alto riesgo médico, los que consumían sustancias adictivas o con inestabilidad domiciliaria, con antecedentes de alergia o paciente con antecedentes de atopía o cualquier otra situación médica que a juicio del investigador fue considerada inoportuna o inapropiada su inclusión. Se consideró como criterio de salida a los pacientes que interrumpieron el tratamiento o dejaron de asistir a las evaluaciones clínicas.

A los pacientes que accedieron a formar parte de la investigación, se les solicitó que cumplimenten con la lectura total del consentimiento informado, luego de consentir por escrito mediante el llenado y rubrica del mismo; posteriormente fueron registrados en planillas diseñadas para el control y la evolución. 


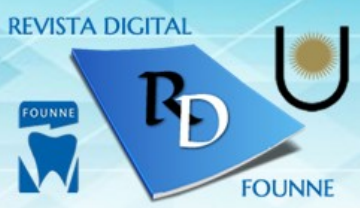

Las evaluaciones del estado inicial se realizaron en ambos grupos, identificado como tiempo 0 , correspondió a la eliminación de la causa, a la toma de muestras para el primer estudio citológico y a la administración de las fórmulas magistrales según corresponda a cada grupo. Luego se implementó el tratamiento, con una posología de dos aplicaciones diarias.

Los controles de la evolución de la lesión bajo del tratamiento se concretaron en dos tiempos operatorios, identificados como tiempo 1 y correspondió al primer control realizado a los 3 días, se complementa con la toma de muestras para el segundo estudio citológico.

El tiempo 2, correspondió al segundo control realizado a los 7 días, a la toma de muestras para el tercer estudio citológico y a la suspensión del tratamiento.

En cada control se consignó el estado de la lesión de acuerdo a las resultantes de la valoración de la sintomatología mediante una escala de Downie modificada ${ }^{26}$ y de los signos mediante una escala milimetrada de la superficie afectada, identificadas como estado 1 (E1) o de leve mejoría, resultante de una reducción en un $25 \%$ de los síntomas y signos registrados en el tiempo 0 ; estado 2 (E2) o de franca mejoría, con una reducción en un 50\% de los síntomas y signos registrados en el tiempo 0; estado 3 (E3) o de cura completa con la remisión en un $100 \%$ de los signos y síntomas y por último el estado 4 (E4) o de persistencia de la lesión.

Se tomaron muestras para citología exfoliativa convencionales, recolectando el material con citobrush, se fijaron en alcohol $96^{\circ}$; posteriormente se colorearon usando el método de Papanicolau. Los parámetros observados al microscopio fueron: celularidad del extendido, evaluando la cantidad de células superficiales e intermedias descamadas, presencia de células parabasales, hematíes, presencia o no de células inflamatorias, polimorfonucleares neutrófilos $(\mathrm{PMN})$, histiocitos y flora microbiana. Se registraron los datos observados en protocolos sistematizados de citología. Se utilizó un mismo observador a los efectos de evitar interpretaciones erróneas. En base a esta identificación se pudo abordar a los siguientes diagnósticos citológicos especificando, con inflamación (Dc1) y sin inflamación (Dc2).

Se realizaron exámenes estomatológicos a 81 pacientes, a 41 se los incluyó en el grupo experimental tratados con la formulación objeto de estudio, tintura de propóleos al 5\% (T.p.) y a 40 se los incluyó en el grupo control tratados con la medicación tradicional (M.t.) (betametasona valerato a $0,1 \%$ ) 


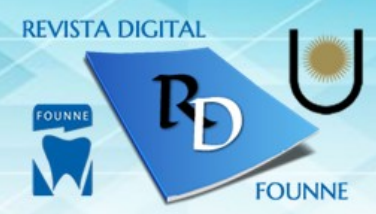

\section{Artículo de Investigación}

\section{Resultados:}

Los controles de la evolución de los tratamientos realizados en los 41 pacientes del grupo experimental tratados con T.p. permitieron registrar que en el tiempo 0, la citología exfoliativa de las lesiones con un resultado de Dc1 en el 100\% de los casos. En el Tiempo 1, el 76\% (31) de los pacientes lograron un estado de leve mejoría y el 24\% (10) de los pacientes alcanzaron un estado de franca mejoría. En el Tiempo 2, el 76\% (31) de los pacientes lograron un estado de cura completa y el $24 \%$ (10) un estado de franca mejoría. (Figura I)

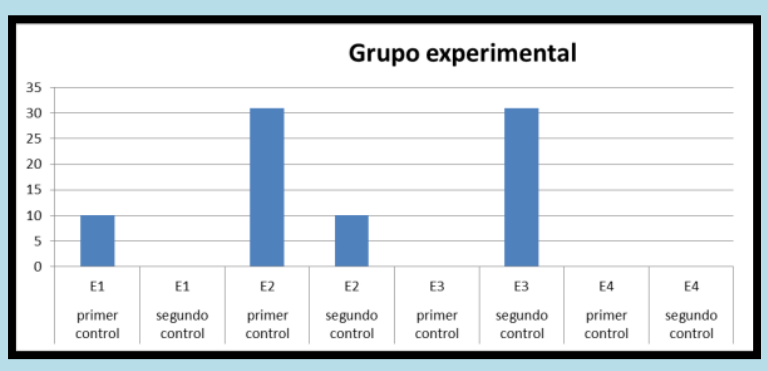

Figura I: Estadios evolutivos del grupo experimental.

La citología exfoliativa realizada al grupo experimental en el tiempo 0 y en el tiempo 1 registró Dc1 en el $100 \%$ los casos. Después de la administración de las formulaciones (T2 - Cex. 3) dio como resultado que en los pacientes del grupo experimental en 11 casos se obtuvo una citología Dc1 y 30 casos logró una citología Dc2. (Figura II)

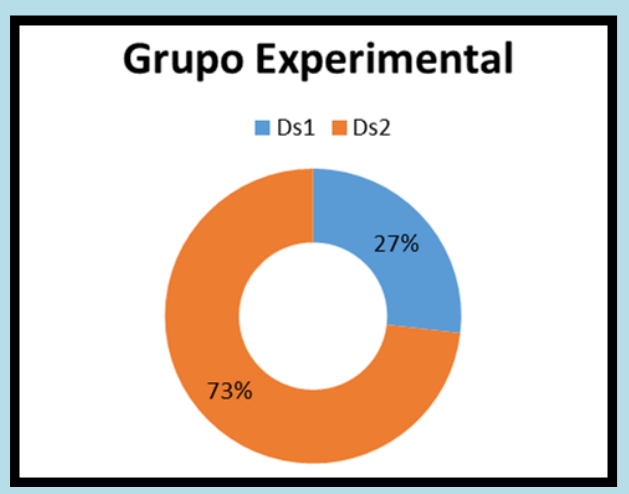

Figura II: Citología exfoliativa del grupo experimental. 


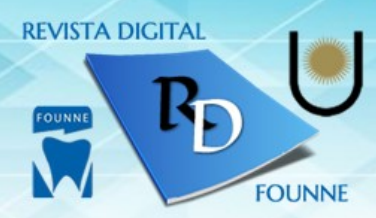

Los controles de la evolución realizados en los 40 pacientes del grupo control tratados con M.t. registró en el tiempo 0, un resultado de Dc1 en el 100\% de los casos. En el Tiempo 1, el 35\% (14) de los pacientes lograron un estado de leve mejoría y el 57,5\% (23) de los pacientes alcanzaron un estado de franca mejoría y en 7, 5 \% (3) pacientes hubo persistencia de la lesión. En el Tiempo 2, el 42,5\% (17) de los pacientes lograron un estado de franca mejoría y el 57,5\% (23) un estado de cura completa. (Figura III)

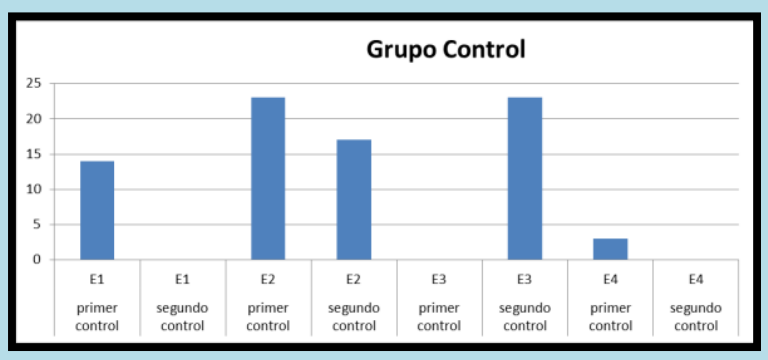

Figura III: Estadios evolutivos del grupo control.

La citología exfoliativa realizada en el grupo control en el tiempo 0 y en el tiempo 1 registró Dc1 en el 100\% los casos (40 pacientes) al igual que el grupo experimental. Después de la administración de las formulaciones (T2 - Cex 3) dio como resultado que en los pacientes del grupo control en 17 casos se obtuvo una citología Dc1 y 33 casos logró una citología Dc2. (Figura IV)

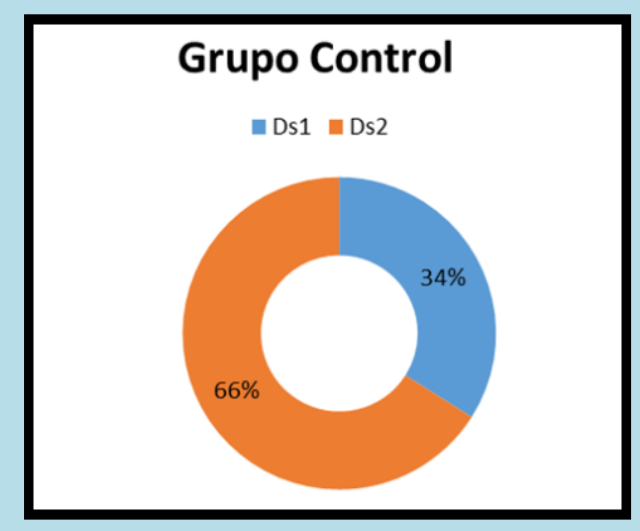

Figura IV: Citología Exfoliativa del grupo control. 


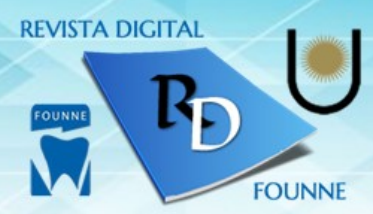

La comparación de las evoluciones de ambos grupos en el primer control a los 3 días demuestra que la medicación experimental logró un mejor resultado ya que el $100 \%$ de los pacientes alcanzaron un estado de franca mejoría y leve mejoría. En comparación con el grupo control que el 92,5\% alcanzó un estado de franca mejoría y leve mejoría; con persistencia de la lesión en un 7,5\%. (Figura V)

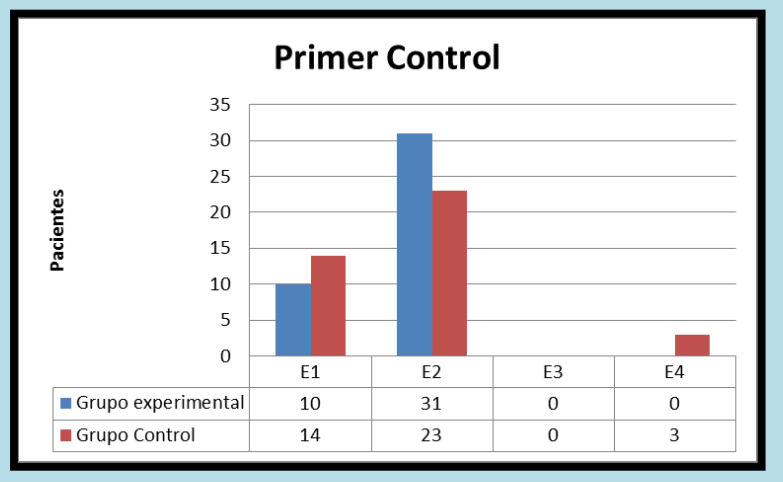

Figura V: Comparación de las evoluciones. Primer control

La comparación de las evoluciones de ambos grupos en el segundo control a los 7 días permitió obtener el resultado de que en ambos grupos se lograron estadios de cura completa y franca mejoría en el $100 \%$ de los casos. (Figura VI)

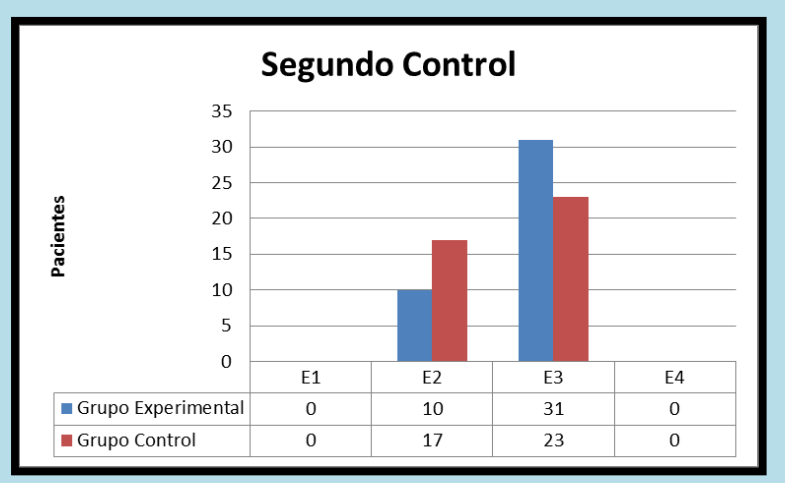

Figura VI: Comparación de las evoluciones. Segundo control.

La comparación de los estadios evolutivos de ambos grupos reveló una supremacía del grupo experimental ( $76 \%$ de cura completa y $24 \%$ de franca mejoría), sobre el grupo control (57,5\% de cura completa y el $42,5 \%$ de franca mejoría) (Figura VII) 


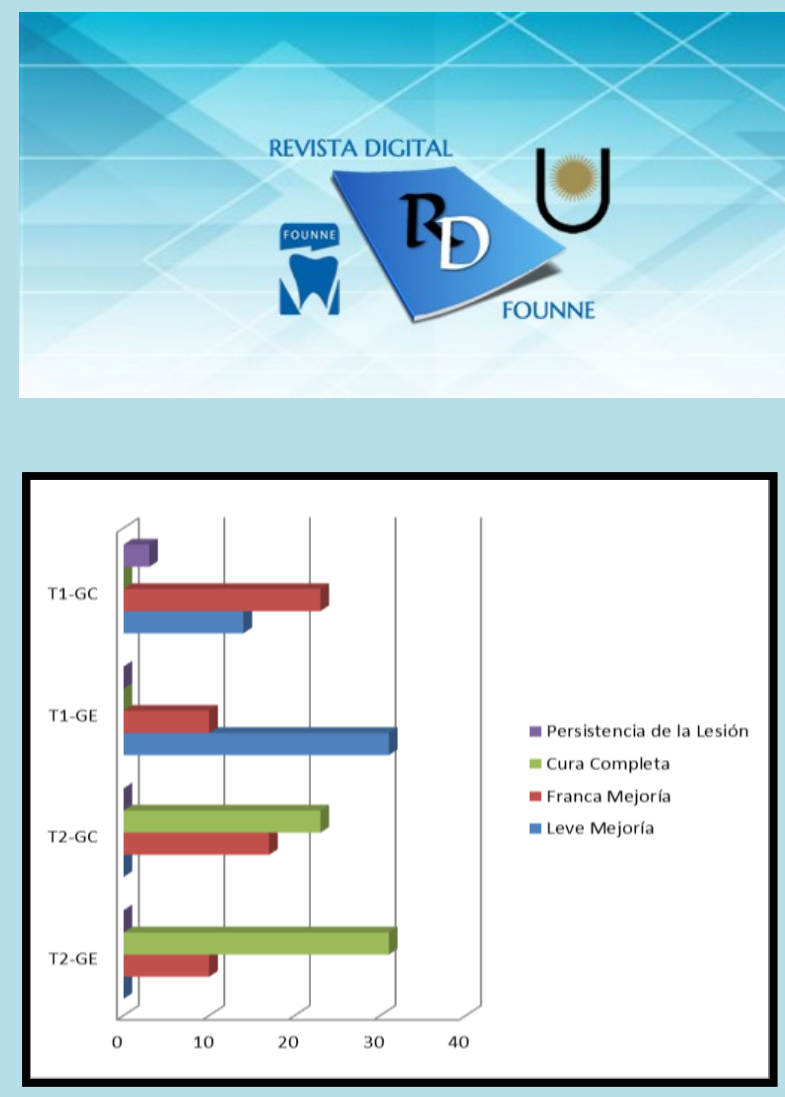

Figura VII: Comparación de los estadios evolutivos.

La evaluación de las diferencias observadas se realizó mediante la utilización de la Prueba de Wilcoxon para muestras independientes, siendo el propósito determinar si los resultados obtenidos en ambos grupos eran estadísticamente significativos.

El análisis estadístico que arrojó un p. valor de 0,0858 estadísticamente no significativas pero clínicamente efectivas, ya que se logró la cura completa de las lesiones en el grupo experimental en el 76\% de los casos (31) y de franca mejoría en el 25\% de los casos (11) en comparación con el grupo control, donde se registró en el 57,5\% (23) un estado de cura completa y en el 42,5\% (17) de los pacientes lograron un estado de franca mejoría. Los resultados obtenidos dan cuenta de una preponderancia cuantitativa de la formulación objeto de estudio en comparación con la medicación tradicional.

La muestra para citología exfoliativa (Cex 3) mostró diferentes porcentajes de Dc1 y Dc2 en el grupo experimental y en el grupo control aunque estadísticamente no fueron significativas ( $\mathrm{p}$ valor $=0.1382)($ Figura IX) 


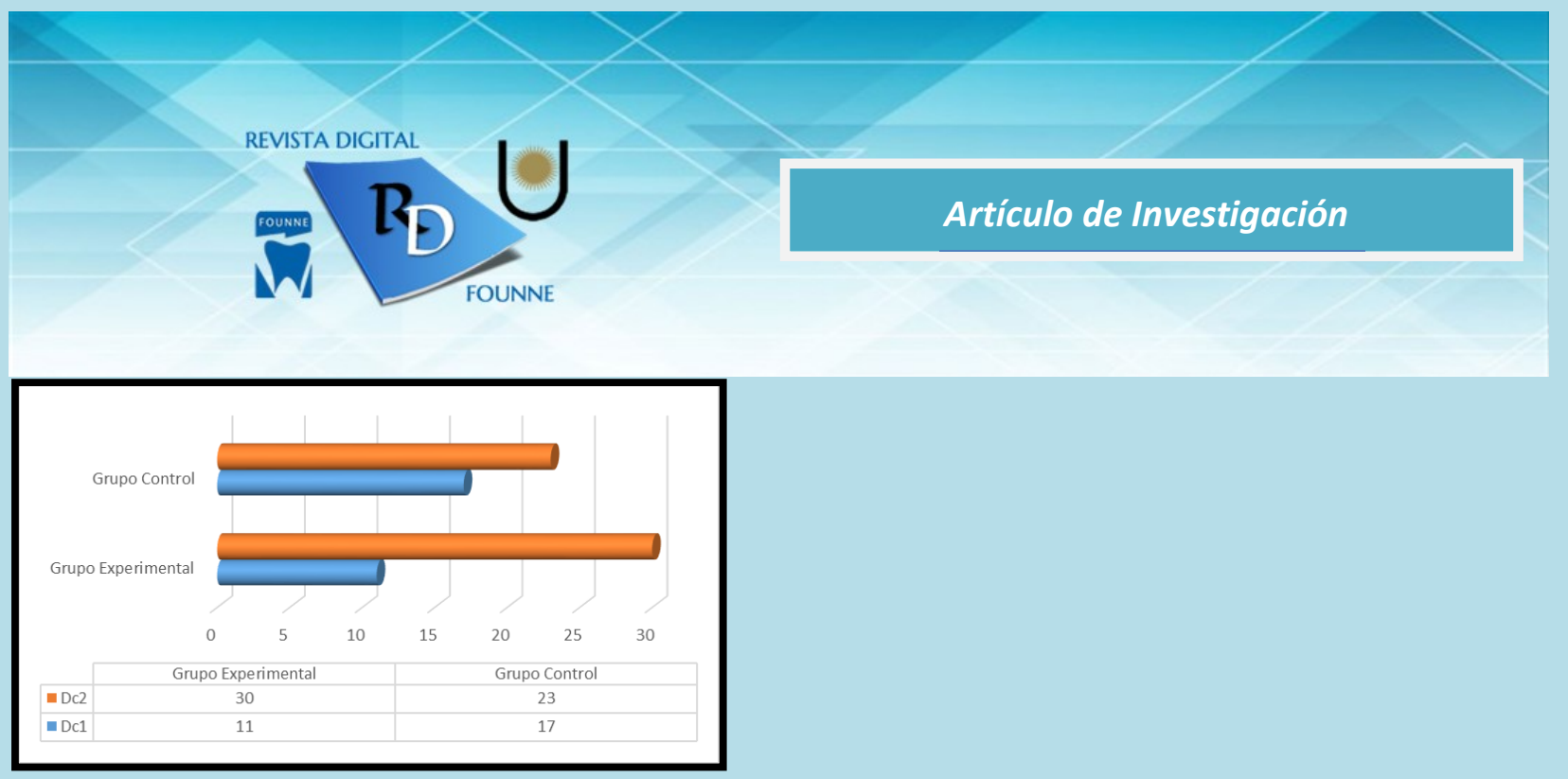

Figura IX: Comparación de la citología exfoliativa final.

\section{Discusión}

La acción de las noxas externas productoras de las queilitis comisurales registradas en las historias clínicas de ambos grupos presentan similares manifestaciones clínicas a las descritas Ceccotti y Sforza. ${ }^{1}$ La respuesta inflamatoria comisural se debe a la perdida de la dimensión vertical oclusiva o subnaso - submentoniana según García López y particularmente en pacientes desdentados portadores de prótesis dentales desadaptadas. ${ }^{2}$

Park, Brodell y Helms sostienen que en la lesión comisural se puede agregar una colonización micótica oportunista del tipo de cándida albicans, caracterizado por una sustancia de color blanquecino, cremoso, que al frote con una gasa deja ver una superficie con múltiples fisuras acompañada de sintomatología dolorosa. ${ }^{4}$

Las propiedades antimicóticas de los propóleos han sido presentadas por Herrera y Alvear en estudios in vitro de extractos de propóleos de diferentes orígenes, registran actividades antifúngicas sobre dermatofitos y especies de cándida. Esta cualidad de destruir hongos depende del origen del propóleos y del solvente usado para su extracción. ${ }^{20 .}$ Se coincide con diferentes autores y entre ellos Premoli y Herrera quienes afirman que la tintura del propóleos, utilizada como fitofármaco asociado a diversos efectos farmacológicos tales como acción antimicrobiana, analgésica, antiinflamatoria, antioxidante y cicatrizante. ${ }^{19}$.

Coincidimos con Ferreira y Torres que ratifican los efectos benéficos del propóleos en la terapéutica de la alveolitis y lesiones periodontales, además se han observado efectos beneficiosos de los propóleos sobre lesiones gingivales, ulceras, aftas bucales y como regenerador de los epitelios en las queilitis angulares. ${ }^{14}$ 


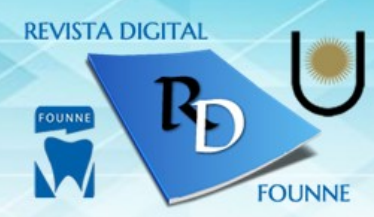

\section{Conclusiones}

Se obtuvieron resultados satisfactorios en la terapéutica de la queilitis comisural a partir de la aplicación de la tintura de propóleos al $5 \%$, reconociendo la efectividad de la formulación magistral. Estos dan cuenta de la reducción de los síntomas y de los signos, con ausencia de reacciones adversas.

La formulación es de aplicación tópica y carente de actividad intrínseca, siendo de administración rápida y de fácil realización. Su aplicación demostró ser efectiva en la remisión del cuadro clínico, favoreciendo la restitución a la normalidad anatómica y funcional del sector afectado.

\section{Bibliografía.}

1. Ceccotti E, Sforza R. El Diagnóstico en Clínica Estomatológica. Editorial Panamericana. Bs. As. Argentina. 2007. 300-302.

2. García López E, Blanco Ruiz A, Rodríguez García L, Reyes Fundora D, et al. Queilitis: Revisión bibliográfica. Rev Cubana Estomatol [revista en la Internet]. 2004 Ago [citado 2016 Jun 28] ; 41(2): . Disponible en: http://scielo.sld.cu/scielo.php?script=sci_arttext\&pid=S003475072004000200009\&lng=es.

3. Bascones Martinez A, García García V, Meurman J, Requena Caballero L. Immunemediated diseases: what can be found in the oral cavity? Int J Dermatol, (2015) 54: 258270. doi:10.1111/ijd.12681

4. Park K, Brodell R, Helms S. Angular cheilitis, part 2: nutritional, systemic, and drugrelated causes and treatment. Cutis. 2011; 88:27-32.

5. Reis Thayane R, Nogueira Brenna M, Domínguez M. Manifestaciones Orales en Pacientes Reumatológicos: una Revisión de los Conocimientos. Int. J. Odontostomat. [Internet]. 2015 Dic [citado 2017 Mayo 09]; 9(3): 413-418. Disponible en:

http://www.scielo.cl/scielo.php?script=sci arttext\&pid=S0718-381X2015000300010\&lng=es http://www.scielo.cl/scielo.php?script=sci_arttext\&pid=S0718-381X2015000300010\&lng=en\&nrm=iso\&tlng=en 


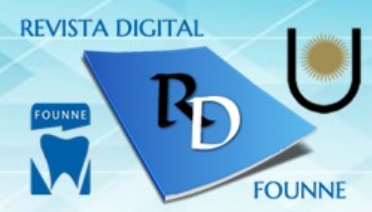

6. Nápoles González I, Rivero Pérez O, García Nápoles C, Pérez Sarduy D. Lesiones de la mucosa bucal asociadas al uso de prótesis totales en pacientes geriatras. AMC [Internet]. $2016 \mathrm{Abr}$ [citado 2017 Mayo 08]; 20(2): 158-166. Disponible en: http://scielo.sld.cu/scielo.php?script=sci_arttext\&pid=S1025-02552016000200008\&lng=es.

7. García Alpizar B, Benet Rodíguez M, Castillo Betancourt E. Prótesis dentales y lesiones mucosas en el adulto mayor: una preocupación de todos. MediSur [Internet]. 2010 Feb [citado 2017 Mayo 10] ; 8( 1 ): 36-41. Disponible en: http://scielo.sld.cu/scielo.php?script=sci_arttext\&pid=S1727-897X2010000100008\&lng=es

8. Gutiérrez Segura M, Sánchez Ramírez Y, Castillo Santiesteban Y. Lesiones en la mucosa oral de pacientes mayores de 60 años y portadores de prótesis. CCM [Internet]. 2013 Dic [citado 2017 Mayo 10] ; 17( 4 ): 452-460. Disponible en: http://scielo.sld.cu/scielo.php?script=sci_arttext\&pid=S1560-43812013000400005\&lng=es

9. García Alpízar B, Capote Valladares M, Morales Montes de Oca T. Prótesis totales y lesiones bucales en adultos mayores institucionalizados. Rev Finlay [Internet]. 2012 [citado 2015 feb 24]; 2(1): [aprox. 12 p.]. Disponible en:

10. Sharon V, Fazel N. Oral candidiasis and angular cheilitis. Dermatol Ther. 2010; 23:230242.

11. Rivas A. Estudio descriptivo de dermatitis de contacto por cosméticos en Medellín, Colombia. Rev. Asoc. Colomb. Dermatol. Cir. Dermatol, 2011, vol. 19, no 4.

12. Arribas M, Soro P, Silvestre J. Dermatitis de contacto alérgica por fragancias. Parte II. Actas Dermo-Sifiliográficas, 2013, vol. 104, no 1, p. 29-37.

13. Bellon Leyva S, Calzadilla Mesa X. Efectividad del uso del propóleos en el tratamiento de la estomatitis aftosa. Rev Cubana Estomatol [online]. 2007, vol.44, n.3 [citado 201704-28], pp. 0-0. Disponible en:

http://scielo.sld.cu/scielo.php?script=sci_arttext\&pid=S0034-75072007000300008\&lng=es\&nrm=iso ISSN 1561-297X.

14. Ferreira F, Torres S, Rosa O, Ferreira C, et al. Antimicrobial effect of propolis and other substances against selected endodontic pathogens. Oral Surg Oral Med Oral Pathol Oral Radiol Endod. 2007; 104(5): 709-16. 


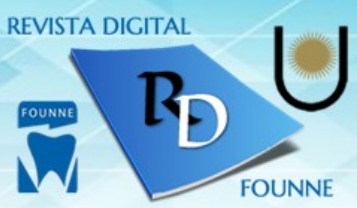

15. Fernández F, Días A, Ramos C, Ikegaki M, et al. The in vitro antifungical activity evaluation of propolis G12 ethanol extracts on Cryptococus neoformans". Rev. Inst. Med. Trop. S. Paulo, 2007. 49(2):93-95.

16. García González A, Castro Martínez J, González Quijano H. Empleo de plantas medicinales en las afecciones estomatológicas por los estudiantes en sus prácticas preprofesionales. Medicentro 2008;12(4)

17. Fernández V, Rosende R, Ortiz Barreto E. Análisis comparativo de dos medicaciones en el tratamiento de las queilitis" Revista de la Facultad de Odontología de la Universidad Nacional del Nordeste. Argentina. 2009. Vol. II. N². p. 20-23.

18. Fernández V, Rosende R, Ortiz Barreto E. Vallejos, R. Acción del óleo calcáreo en el tratamiento de las queilitis por contacto crónicas. Revista de la Facultad de Odontología de la Universidad Nacional del Nordeste. Argentina. 2013. Vol. VI. No2. p. 7-12.

19. Premoli G, Laguado P, Díaz N, Romero C, et al. Uso del Propóleos en Odontología. Acta Odontológica Venezolana 2010. Vol $48 \mathrm{~N}^{\mathrm{o}} 2$

20. Herrera C, Alvear M, Barrientos L, Montenegro G, et al. The antifungal effect of six commercial extracts of Chilean propolis on Candida spp. Cien. Inv. Agr. 2010. 37(1):7584.

21. Noriega Salmón V. El propóleos, otro recurso terapéutico en la práctica clínica. 2014.

22. Moromi H, Martínez Cadillo E, Ramos Perfecto D. Antibacterianos naturales orales: Estudios en la Facultad de Odontología de la Universidad Nacional Mayor de San Marcos. Odontología Sanmarquina, 2014, vol. 12, no 1, p. 25-28.

23. Solomon D, Davey D. Kurman R. Moriarty A. et al. The 2001 Bethesda System. JAMA, 287: 2114, 2002.

24. Ruiz Salvador A, García Milián A, Jiménez López G, Alfonso Orta I. Farmacovigilancia de fitofármacos y apifármacos en Cuba durante 2006-2010 Revista Cubana de Plantas Medicinales. 2013;18(2): 173-186

25. Santos Muñoz L, Perdomo Delgado J, González Pla E. Comportamiento de las reacciones adversas reportadas por productos naturales. Matanza 2003-2008.Revista Médica Electrónica 2009;31(6)

26. Downie W.Studies with pain rating scales. Ann Rheum Dis 1979; 37: 378-81. 